\title{
MicroRNA-665 promotes the proliferation of ovarian cancer cells by targeting SRCIN1
}

\author{
PING ZHOU, TINGCHUAN XIONG, LILI YAO and JIANLIN YUAN \\ Department of Gynecology and Obstetrics, Cancer Hospital Affiliated to Xinjiang Medical University, \\ Ürümqi, Xinjiang 830011, P.R. China
}

Received February 20, 2019; Accepted November 8, 2019

DOI: $10.3892 /$ etm.2019.8293

\begin{abstract}
Recent studies have discovered several microRNAs (miRNAs/miRs) as biomarkers for the prediction of ovarian cancer by detecting miRNA profiles in serum samples from healthy volunteers and patients with ovarian cancer. However, whether and how these miRNAs are involved in tumorigenesis is not known. In the present study, the expression of miR-665, a recently discovered biomarker for ovarian cancer, was upregulated in tumor tissues from patients with ovarian cancer compared with normal tissues. Inhibition of miR-665 inhibited cell proliferation ability and inactivated MAPK/ERK signaling of ovarian cancer cells. Using bioinformatics analysis, Src kinase signaling inhibitor 1 (SRCIN1) was predicted as a potential target gene of miR-665. Reverse transcription-quantitative PCR and western blotting showed that SRCIN1 expression was repressed by miR-665 in ovarian cancer cells. In addition, a dual luciferase activity assay showed that SRCIN1 was a target gene of miR-665. Silencing of SRCIN1 could reverse the cell growth arrest, which was induced by the miR-665 inhibitor. Moreover, miR-665 levels were negatively correlated with SRCIN1 mRNA levels in tumor tissues from patients with ovarian cancer. In conclusion, the present data suggested that miR-665 functioned as an oncogene in ovarian cancer by directly repressing the expression of SRCIN1.
\end{abstract}

\section{Introduction}

According to the global cancer statistics in 2018, ovarian cancer was the 8th most diagnosed cancer type and a leading cause of cancer-related death for women worldwide (1). Surgical removal and chemotherapy are two current approaches to treat

Correspondence to: Dr Jianlin Yuan, Department of Gynecology and Obstetrics, Cancer Hospital Affiliated to Xinjiang Medical University, 789 Suzhoudong Street, Ürümqi, Xinjiang 830011, P.R. China

E-mail: yuanjianlindoctor@tom.com

Key words: microRNA-665, Src kinase signaling inhibitor 1, ovarian cancer patients with ovarian cancer; however, the efficacy of these treatments is limited due to the development of drug resistance and recurrence of cancer $(2,3)$. Although numerous previous clinical and experimental studies have provided novel insight into the molecular mechanisms of ovarian cancer, patients with advanced-stages of ovarian cancer still have a poor prognosis $(4,5)$. There is an urgent need to further understand the molecular mechanisms of ovarian cancer for the development of targeted therapeutic strategies.

MicroRNAs (miRNAs/miRs) are small, non-coding, single-stranded nucleotides which are ubiquitously expressed in eukaryotic cells (6). Mechanistically, miRNAs directly bind to the 3'untranslated region (UTR) of their target mRNAs, leading to the degradation of mRNA or inhibition of translation (7). The expression of miRNA is tightly controlled in normal cells, for instance, dysregulation of several key miRNAs resulted in the disruption of cell signaling networks in human diseases, including cancer (8-10). With microarray analysis, many differentially expressed miRNAs are detected between ovarian tumors and normal tissues $(11,12)$. miRNAs are identified as tumor suppressors or oncogenes based on their potential prognostic predictor value (13). For example, the expression level of miR-21 in serum was considered as a biomarker for the early detection and prediction of prognosis for patients with ovarian cancer in 2013 (14). Later experimental studies demonstrated that miR-21 modulates drug sensitivity by targeting several key genes $(15,16)$. Most recently, patients have been accurately diagnosed with ovarian cancer from the expression of 10 miRNAs, which is deemed as a diagnostic model (17). However, how these miRNAs contribute to the progression of ovarian cancer is unknown.

The v-src avian sarcoma (Schmidt-Ruppin A-2) viral oncogene homolog ( $\mathrm{Src}$ ) is a well-characterized oncogenic tyrosine kinase that is frequently upregulated in cancer (18). In ovarian cancer, activation of the Src signaling pathway is crucial for the epithelial-mesenchymal transition (EMT) process and in vivo tumor growth in nude mice (19). Previous studies have demonstrated that Src is associated with the activity of MAPK/ERK signaling and PI3K/AKT signaling in cancer cells, which are pivotal for cell proliferation and survival $(20,21)$. Src kinase signaling inhibitor 1 (SRCIN1) functions as a tumor suppressor via inactivation of Src in cancer (22).

In the present study, miR-665 levels were upregulated in tumor tissues from patients with ovarian cancer compared 
with normal tissues. Inhibition of miR-665 inhibited cell proliferation and colony forming ability of ovarian cancer cells. SRCIN1 was predicted and validated as a target gene of miR-665. Silencing of SRCIN1 could reverse the miR-665 inhibitor-induced cell growth arrest. Moreover, miR-665 levels were negatively correlated with SRCIN1 mRNA levels in tumor tissues from patients with ovarian cancer. In conclusion, the present data suggested that miR-665 functioned as an oncogene in ovarian cancer by directly repressing the expression of SRCIN1. The present results may provide novel insight into clinically relevant treatments for ovarian cancer.

\section{Materials and methods}

Collection of tumor and normal tissues. In total, 40 pairs of tumor tissues and normal tissues were collected from female patients (aged from 29 to 71 years, with a median age of 53 years) with ovarian cancer who underwent surgery at The Cancer Hospital Affiliated to Xinjiang Medical University during June 2015 to July 2018. Written consent was provided by all the participants before enrollment in the present study. Patients who received chemotherapy or radiotherapy prior to surgery were excluded. The Ethic Committee of Xinjiang Medical University approved the present study. The tumor tissues and normal tissues were collected during surgery removal, and were immediately snap-frozen in liquid nitrogen before RNA extraction and reverse transcription-quantitative PCR (RT-qPCR) were performed.

Cell culture. The human ovarian cancer cell lines SKOV3 and ES2 were purchased from The Type Culture Collection of The Chinese Academy of Sciences. All cell lines were cultured in RPMI-1640 medium (Gibco; Thermo Fisher Scientific, Inc.) supplemented with $10 \%$ FBS (HyClone; GE Healthcare Life Sciences) in a humidified incubator with $5 \% \mathrm{CO}_{2}$. A normal human ovarian surface epithelial (HOSE) cell line was established by following a previously reported method (23). Fresh ovarian scrapings obtained from patients during the surgery described above were immortalized with human papilloma virus 16 E6/E7 oncogenes. The cells were maintained in mammary epithelial cell growth medium (BulletKit ${ }^{\mathrm{TM}}$; Clonetics; Lonza Group, Ltd.) supplemented with 1\% FBS (HyClone; GE Healthcare Life Sciences).

$R N A$ extraction and $R T-q P C R$. Total RNA was extracted from the tissues of the patients and SKOV3 and ES2 cells using TRIzol ${ }^{\circledR}$ reagent (Invitrogen; Thermo Fisher Scientific, Inc.) following the manufacturer's protocol. RNA was reverse transcribed to first-stranded cDNA with PrimeScript ${ }^{\mathrm{TM}}$ First Strand cDNA Synthesis kit (Takara Bio, Inc.). The reverse transcription conditions were as follows: $37^{\circ} \mathrm{C}$ for $15 \mathrm{~min}$ and $85^{\circ} \mathrm{C}$ for 5 sec. RT-qPCR was performed with SYBR Premix Ex Taq (Takara Bio, Inc.) on a CFX96 Touch Real-time PCR Detection System (Bio-Rad Laboratories, Inc.). The thermocycling conditions were as follows: $95^{\circ} \mathrm{C}$ for $30 \mathrm{sec}$, followed by 35 cycles of $95^{\circ} \mathrm{C}$ for $5 \mathrm{sec}$ and $60^{\circ} \mathrm{C}$ for $30 \mathrm{sec}$. U6 and GAPDH served as internal controls for miRNA and mRNA, respectively. The relative expression of genes was calculated using the $2^{-\Delta \Delta \mathrm{Cq}}$ method (24). The primer sequences were as follows: SRCIN1-forward: 5'-GAGGCTCGCAACGTCTTCTAC-3'; SRCIN1-reverse: 5'-GCGATGCGTACACCATCTCTC-3'; GAPDH-forward: 5'-GGAGCGAGATCCCTCCAAAAT-3'; GAPDH-reverse: 5'-GGCTGTTGTCATACTTCTCATGG-3'; stem-loop primer: 5'-CTCAACTGGTGTCGTGGAGTC GGCAATTCAGTTGAGAGGGGCC-3'; miR-665-forward: 5'-GCCGAGACCAGGAGGCUGA-3'; miR-665-reverse: 5'-CTCAACTGGTGTCGTGGA-3'; U6-forward: 5'-GCTTCG GCAGCACATATACTAAAAT-3'; and U6-reverse: 5'-CGC TTCACGAATTTGCGTGTCAT-3'.

Downregulation and upregulation of miR-665 in ovarian cancer cells. miR-665 inhibitor (5'-AGGGGCCUCAGCCUC CUGGU-3'), miR-665 mimic (5'-ACCAGGAGGCUGAGG CCCCU-3') and the corresponding negative controls (miR-NC; 5'-UCGCUUGGUGCAGGUCGGGAA-3') were synthesized and purchased from Shanghai GenePharma Co., Ltd. SKOV3 and ES2 cells were seeded into each well of 24-well plates ( $2 \times 10^{5}$ cells per well) and were transfected with miR-665 inhibitor, miR-665 mimic, miR-NC inhibitor or miR-NC mimic at a concentration of $50 \mathrm{nM}$ with Lipofectamine ${ }^{\circledR} 3000$ (Invitrogen; Thermo Fisher Scientific, Inc.) following the manufacturer's protocol, and maintained for $48 \mathrm{~h}$ before any subsequent experiments were performed.

Silencing of SRCIN1 in ovarian cancer cells. Control siRNA and SRCIN1 siRNA were synthesized and purchased from Shanghai GenePharma Co., Ltd. The sequences were as follows: Control siRNA sequence: 5'-UUCUCCGAACGU GUCACGUTT-3'; and SRCIN1 siRNA sequence: 5'-CGGGAG AGAGGCAGGCUCUGUCGGAATT-3'. For the silencing of SRCIN1, $50 \mathrm{nM}$ SRCIN1 siRNA was transfected into SKOV3 and ES2 cells in 24-well plates $\left(2 \times 10^{5}\right.$ cells per well) using Lipofectamine ${ }^{\circledR}$ RNAiMax (Invitrogen; Thermo Fisher Scientific, Inc.) following the manufacturer's protocol. After $48 \mathrm{~h}$, the cells were collected for western blotting.

Western blotting. SRCIN1 (cat. no. ab244527; 1:1,000) and GAPDH (cat. no. ab8245; 1:5,000) antibodies were bought from Abcam. AKT (cat. no. 4685; 1:1,000), phosphorylated (p)-AKT (cat. no. 4060; 1:1,000), ERK1/2 (cat. no. 4695; $1: 1,000$ ) and p-ERK1/2 (cat. no. 4370; 1:1,000) primary antibodies were purchased from Cell Signaling Technology, Inc. HRP-conjugated secondary antibodies against rabbit (cat. no. SA00001-2; 1:10,000) and mouse (cat. no. SA00001-1; 1:10,000) were products of ProteinTech Group, Inc. Protein lysates were prepared from cells with RIPA lysis buffer (Sigma-Aldrich; Merck KGaA). The protein concentration was detected with a BCA Protein Assay kit (Pierce; Thermo Fisher Scientific, Inc.). For the western blotting, $20 \mu \mathrm{g}$ protein was loaded on $8 \%$ gels and separated by SDS-PAGE. After electrophoresis, the proteins were transferred from SDS-PAGE gels to PVDF membranes. The membranes were then blocked in 5\% non-fat milk at room temperature for $1 \mathrm{~h}$. The membrane was subsequently incubated with the appropriate primary antibody at $4^{\circ} \mathrm{C}$ overnight and the appropriate secondary antibody at room temperature for $2 \mathrm{~h}$. The blots were developed with ECL western blotting substrate (Pierce; Thermo Fisher Scientific, Inc.). The images of blots were 
analyzed using ImageJ software version 1.6.0 (National Institutes of Health).

Cell proliferation assay. To determine the proliferation of cells, a Cell Counting Kit-8 (CCK-8; Dojindo Molecular Technologies, Inc.) was used according to the manufacturer's protocol. In total, 10,000 cells were plated in each well of 96-well plates. At $48 \mathrm{~h}$ after transfection with miR-665 inhibitor or miR-NC inhibitor, $10 \mu \mathrm{l}$ CCK- 8 solution was added into the well and incubated for another $2 \mathrm{~h}$. Afterwards, the medium was transferred to another new 96-well plate, and the absorbance at $450 \mathrm{nM}$ was detected using a microplate reader to detect the cell proliferation.

Colony forming assay. The colony forming assay was performed in a standard procedure. A total of 2,000 cells were plated in each well on 6-well plates. After transfection with miR-665 inhibitor or miR-NC inhibitor with or without control small interfering RNA (siRNA) or SRCIN1 siRNA, the cells were incubated for 10 days to form cell colonies. The culture medium was discarded, and the cells were washed with PBS. The cells were fixed with $4 \%$ paraformaldehyde (Beijing Solarbio Science \& Technology Co., Ltd.) for $1 \mathrm{~h}$ at room temperature. After that, the cell colonies were stained with Crystal Violet Staining Solution (Beyotime Institute of Biotechnology) for $20 \mathrm{~min}$ at room temperature. The staining solution was then discarded and the wells were washed with PBS. Images were captured using an inverted microscope in three random fields for each well (x10). The colony numbers were counted using ImageJ version 1.6.0 (National Institutes of Health).

Dual luciferase reporter assay. The full length of SRCIN1 3'UTR was amplified from SKOV3 cDNA with TransFast ${ }^{\circledR}$ Taq DNA Polymerase (TransGen Biotech Co., Ltd.) and ligated into a pGL3 plasmid (Promega Corporation). The PCR conditions were as follows: $94^{\circ} \mathrm{C}$ for $3 \mathrm{~min}$, followed by 35 cycles of $94^{\circ} \mathrm{C}$ for $5 \mathrm{sec} ; 55^{\circ} \mathrm{C}$ for $15 \mathrm{sec}$ and $72^{\circ} \mathrm{C}$ for $10 \mathrm{sec}$. The primer sequences were: SRCIN1-forward: $5^{\prime}$-CTCTAGAAA GCCCCTCACCCCGCTG-3'; SRCIN1-reverse: 5'-CTCTAG AGAAGGAGAUCCAGGAGAG-3'. A total of three site mutations were introduced into the pGL3-SRCIN1-wild-type (WT) plasmid to establish the pGL3-SRCIN1-mutant (Mut). miR-665 mimic or miR-NC $(50 \mathrm{nM})$ in combination with $2 \mu \mathrm{g}$ pGL3-SRCIN1-WT or pGL3-SRCIN1-Mut were transfected into cells with Lipofectamine ${ }^{\circledR} 3000$ and incubated for $48 \mathrm{~h}$. The relative luciferase activity was detected with the Dual Luciferase Reporter assay system (Promega Corporation) according to the manufacturer's protocol. Firefly luciferase was normalized to Renilla luciferase.

Bioinformatic analysis. The potential target genes of miR-665 were predicted using TargetScan software V7.2 (http://www. targetscan.org/vert_72/).

Statistical analysis. All data were analyzed using GraphPad Prism 5.0 (GraphPad Software, Inc.) and are presented as the mean \pm SD. Differences between two groups were compared with Student's t-test, and differences among three groups were compared with one-way ANOVA, followed by Newman-Keuls analysis. The association between SRCIN1 mRNA levels and miR-665 expression was analyzed with Pearson correlation analysis. $\mathrm{P}<0.05$ was considered to indicate a statistically significant difference. All experiments were performed at least three times.

\section{Results}

miR-665 is upregulated in ovarian cancer tissues and cell lines. To investigate the potential role of miR-665 in ovarian cancer, RT-qPCR was performed to detect the expression level of miR-665 in 40 pairs of tumor and normal tissues from patients with ovarian cancer. miR-665 expression was significantly upregulated in tumor tissues compared with normal tissues (Fig. 1A). SKOV3 and ES2 are well-characterized ovarian cancer cell lines. Both of these commonly used ovarian cancer cell lines originated from ovarian clear cell carcinoma (25). Furthermore, RT-qPCR was used to examine the difference in miR-665 expression level between ovarian cancer cell lines (SKOV3 and ES2) and the immortal ovarian epithelial HOSE cell line. The present results demonstrated that miR-665 was significantly upregulated in SKOV3 and ES2 cells compared with HOSE cells (Fig. 1B).

Downregulation of miR-665 inhibits cell proliferation and colony forming ability of ovarian cancer cells. To study the function of miR-665, miR-665 inhibitor was transfected into SKOV3 and ES2 cells to downregulate the miR-665 level, which was verified by RT-qPCR (Fig. 2A). Downregulation of miR-665 led to significant cell growth arrest in SKOV3 cells (Fig. 2B). Consistently, the miR-665 inhibitor also significantly decreased the cell proliferation ability in ES2 cells (Fig. 2C). In addition, miR-665 inhibition significantly inhibited the colony forming ability of SKOV3 and ES2 cells (Fig. 2D and E). The present data suggested that miR-665 promotes cell proliferation in ovarian cancer cells.

Downregulation of miR-665 inactivates the MAPK/ERK pathway in ovarian cancer cells. Hyperactivation of the MAPK/ERK and the PI3K/AKT signaling pathways plays key roles in uncontrolled cell proliferation of ovarian cancer $(20,21)$. The results of the western blotting showed that downregulation of miR-665 decreased the expression of p-ERK1/2 but not p-AKT (Fig. 3A). Further analysis demonstrated that the p-ERK1/2 to ERK1/2 ratio was significantly decreased in cells transfected with miR-665 inhibitor (Fig. 3B), suggesting the inactivation of MAPK/ERK signaling. However, the p-AKT/AKT ratio was not affected (Fig. 3C). The present data suggested that miR-665 might promote ovarian cancer cell proliferation via activation of MAPK/ERK signaling.

miR-665 represses SRCIN1 expression in ovarian cancer cells. TargetScan was used to predict the potential target genes of miR-665. Among them, the 3'UTR of SRCIN1, a negative regulator of MAPK/ERK signaling (22), was identified to harbor binding sites for miR-665 (Fig. 4A). The western blotting results demonstrated that the downregulation of miR-665 significantly increased the protein expression level of SRCIN1 in SKOV3 and ES2 cells (Fig. 4B and C). Furthermore, the 
A

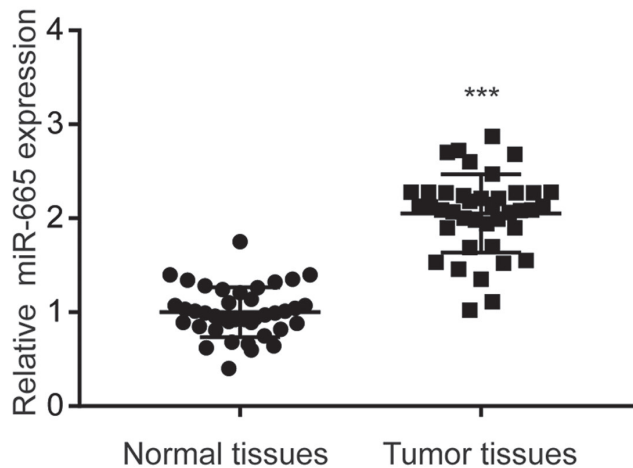

B

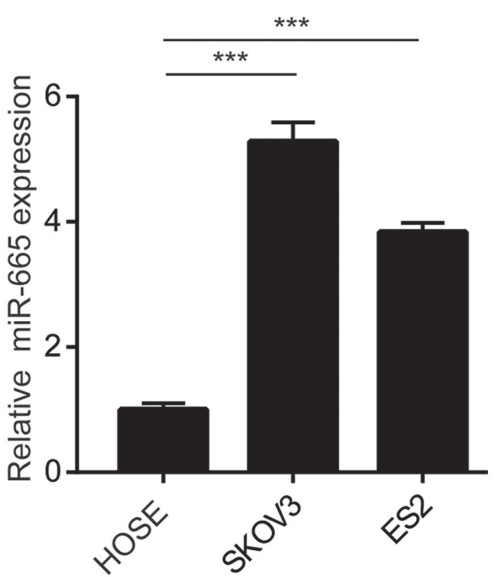

Figure 1. Upregulation of miR-665 in ovarian cancer. (A) miR-665 was upregulated in tumor tissues compared with normal tissues from 40 patients with ovarian cancer. ${ }^{* * *} \mathrm{P}<0.001$ vs. normal tissues. (B) miR-665 was upregulated in ovarian cancer cell lines (SKOV3 and ES2) compared with the immortal HOSE cell line. ${ }^{* * * *} \mathrm{P}<0.001$. miR-665, microRNA-665; HOSE, human ovarian surface epithelial.
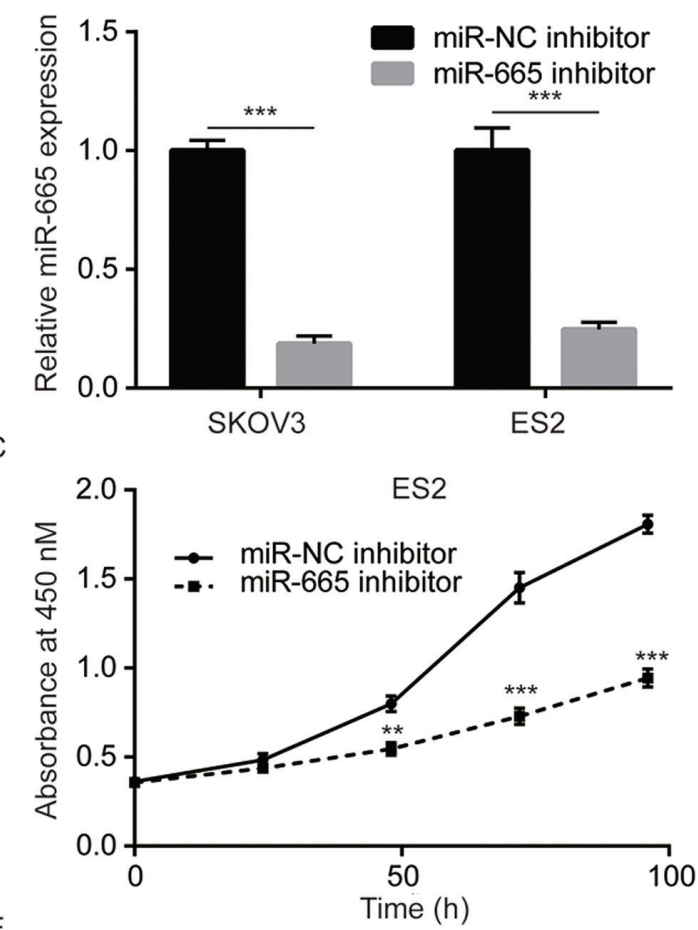

$\mathrm{E}$

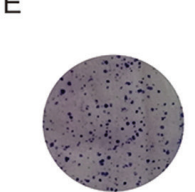

miR-NC inhibitor

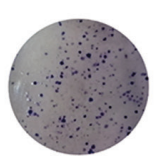

miR-665 inhibitor
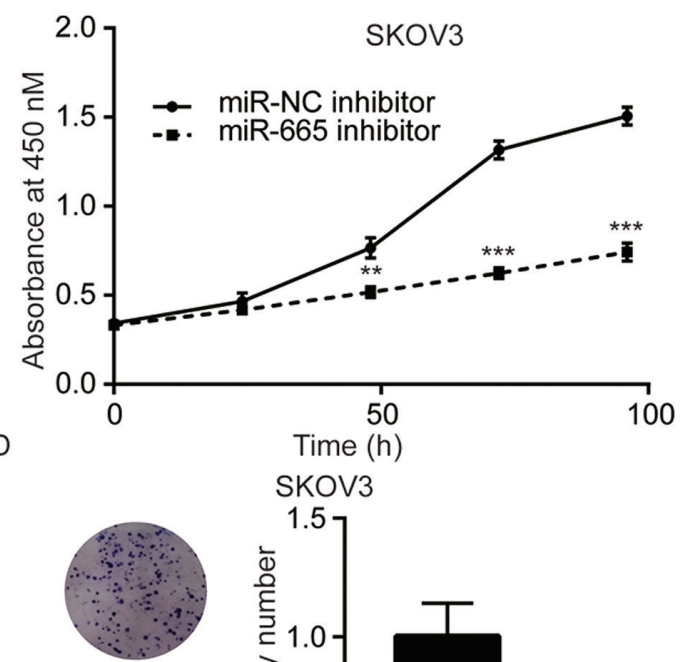

SKOV3

miR-NC inhibitor

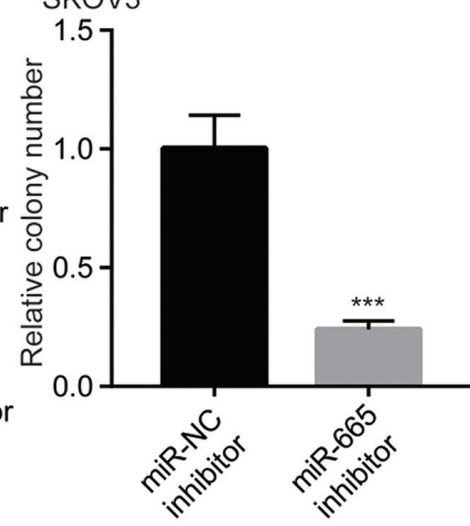

miR-665 inhibitor

Figure 2. Downregulation of miR-665 inhibits cell proliferation in ovarian cancer cells. (A) Transfection of miR-665 inhibitor decreased miR-665 levels in ovarian cancer cell lines (SKOV3 and ES2). (B) miR-665 inhibitor inhibited cell proliferation in SKOV3 cells. (C) miR-665 inhibitor inhibited cell proliferation in ES2 cells. (D) miR-665 inhibitor inhibited colony forming ability in SKOV3 cells. (E) miR-665 inhibitor inhibited colony forming ability in ES2 cells ${ }^{* *} \mathrm{P}<0.01 ;{ }^{* * *} \mathrm{P}<0.001$ vs. miR-NC inhibitor. miR-665, microRNA-665; miR-NC, microRNA negative control. 
A

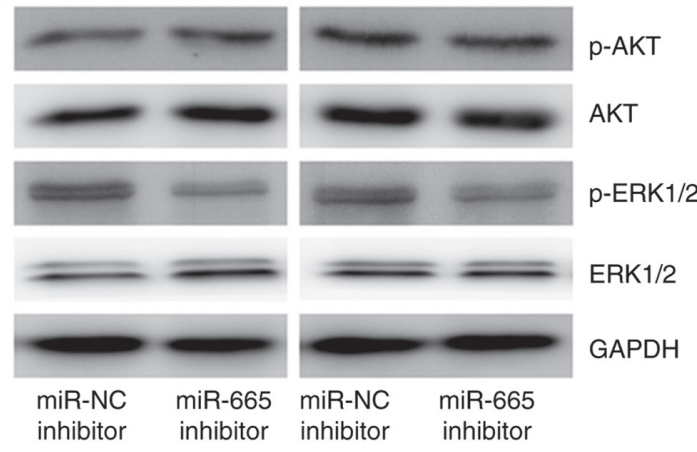

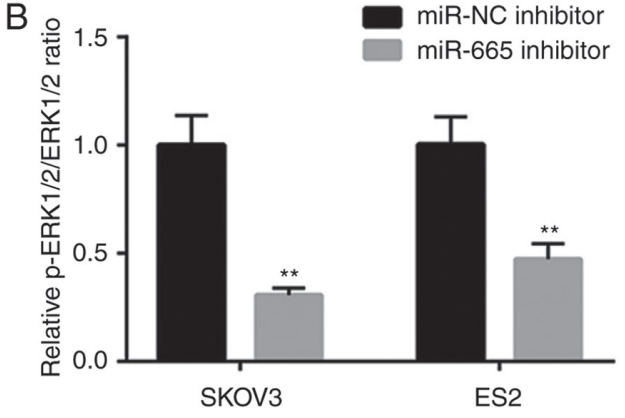

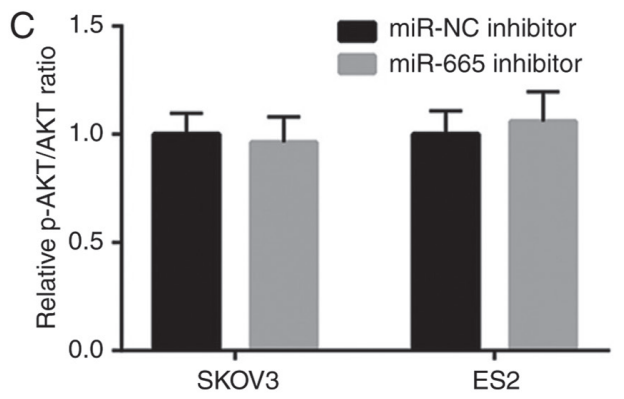

Figure 3. Downregulation of miR-665 inactivates MAPK/ERK signaling in ovarian cancer cells. (A) Western blotting showed that miR-665 inhibitor decreased the protein expression level of p-ERK1/2 but not p-AKT, ERK1/2 or AKT expression in SKOV3 and ES2 cells. (B) Quantification analysis showed that the ratio of p-ERK1/2 to ERK1/2 was decreased with miR-665 downregulation. (C) Quantification analysis showed that the ratio of p-AKT to AKT was not changed with miR-665 downregulation. ${ }^{* *} \mathrm{P}<0.01$ vs. respective miR-NC inhibitor. miR-665, microRNA-665; miR-NC, microRNA negative control; p-, phosphorylated.

A

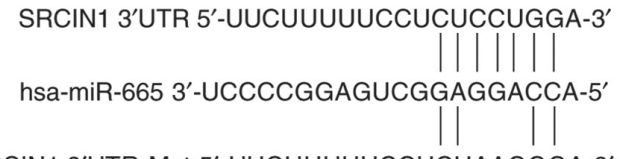

SRCIN1 3'UTR-Mut 5'-UUCUUUUUCCUCUAAGGGA-3'

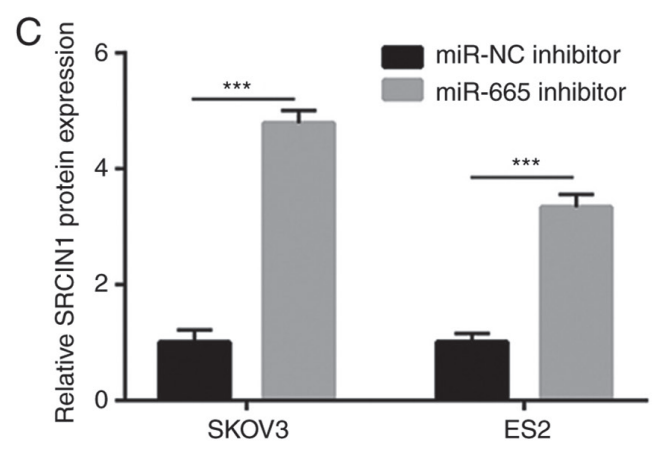

B

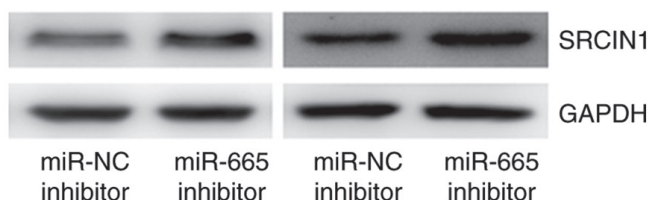

D

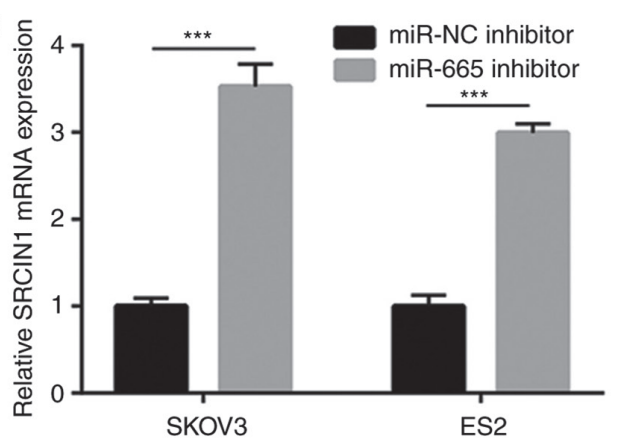

Figure 4. Downregulation of miR-665 increases SRCIN1 expression in ovarian cancer cells. (A) Binding sequence between SRCIN1 and miR-665 is presented. (B) Downregulation of miR-665 increased the protein level of SRCIN1 in SKOV3 and ES2 cells. (C) Quantitative analysis of SRCIN1 expression. (D) Reverse transcription-quantitative PCR showed that the miR-665 inhibitor increased the mRNA expression level of SRCIN1 in SKOV3 and ES2 cells. ${ }^{* * *}$ P $<0.001$. miR-665, microRNA-665; miR-NC, microRNA negative control; SRCIN1, Src kinase signaling inhibitor 1; UTR, untranslated region; Mut, mutant.

results of RT-qPCR demonstrated that miR-665 inhibition significantly increased the mRNA expression level of SRCIN1 in SKOV3 and ES2 cells (Fig. 4D).

SRCIN1 is a target gene of miR-665 in ovarian cancer cells. To further demonstrate SRCIN1 as a target gene of miR-665, a dual luciferase reporter assay was performed. As presented in Fig. 5A, the transfection of miR-665 mimic increased miR-665 expression in ovarian cancer cells. Overexpression of miR-665 reduced the relative luciferase activity of SKOV3 cells that were transfected with SRCIN1 3'UTR-WT (Fig. 5B). A consistent result was observed in ES2 cells (Fig. 5C). The present data demonstrated that SRCIN1 was a target gene of miR-665 in ovarian cancer cells.

miR-665 regulates cell proliferation via regulation of SRCIN1 in ovarian cancer cells. SRCIN1 siRNA was used to study the role of SRCIN1 in miR-665 mediated cell proliferation of 


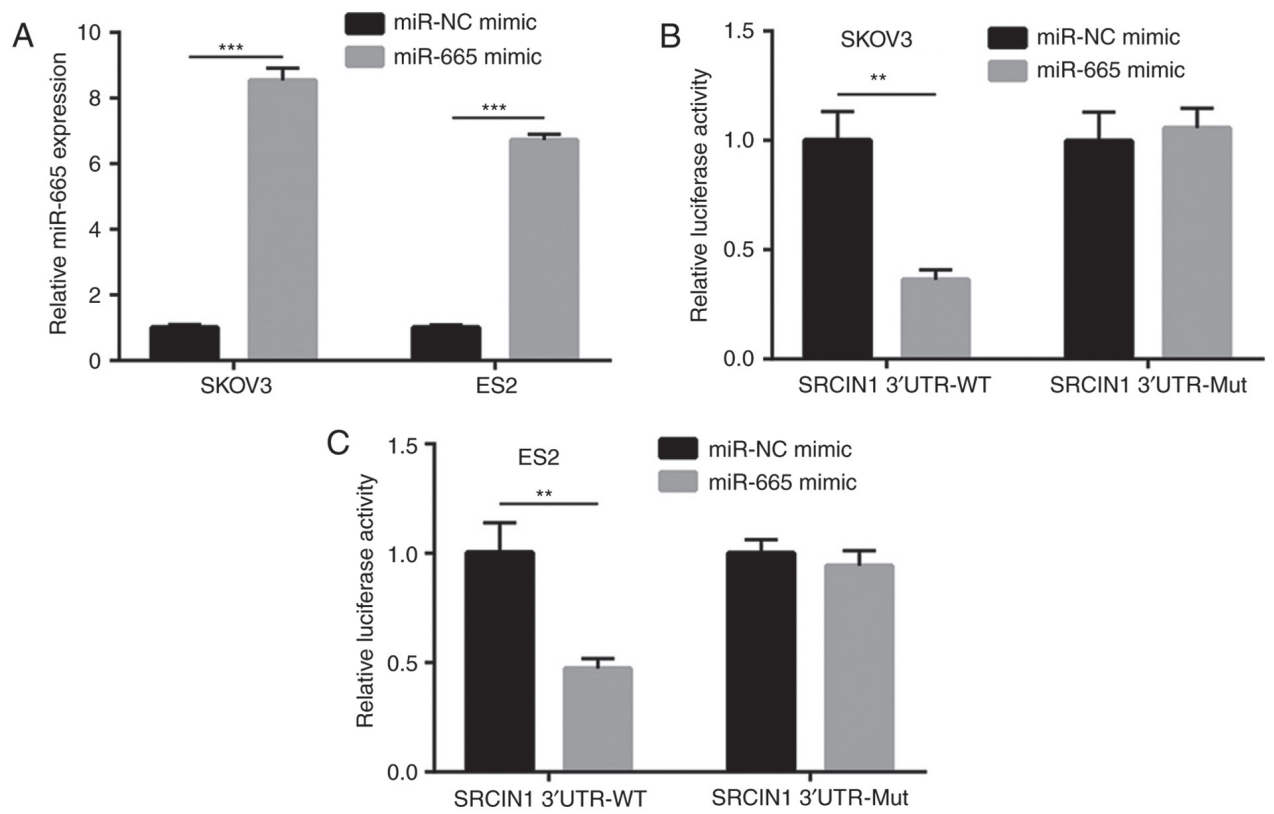

Figure 5. SRCIN1 is a direct target gene of miR-665 in ovarian cancer cells. (A) Transfection of miR-665 mimic increased miR-665 expression in SKOV3 and ES2 cells. (B) miR-665 mimic reduced the relative luciferase activity of SKOV3 cells transfected with SRCIN1 3'UTR-WT compared with SRCIN1 3'UTR-Mut. (C) miR-665 mimic reduced relative luciferase activity in ES2 cells transfected with SRCIN1 3'UTR-WT compared with SRCIN1 3'UTR-Mut. ${ }^{* *} \mathrm{P}<0.01$; ${ }^{* * *} \mathrm{P}<0.001$. miR-665, microRNA-665; miR-NC, microRNA negative control; SRCIN1, Src kinase signaling inhibitor 1; UTR, untranslated region; WT, wild-type; Mut, mutant.

ovarian cancer cells. SRCIN1 siRNA significantly decreased SRCIN1 protein expression in SKOV3 cells (Fig. 6A and B). Additionally, transfection of miR-665 inhibitor significantly increased the SRCIN1 protein level in SKOV3 cells, which was significantly decreased upon transfection of SRCIN1 siRNA (Fig. 6C and D). The cell proliferation assay showed that miR-665 downregulation significantly inhibited cell proliferation of SKOV3 cells, which was reversed after SRCIN1 silencing (Fig. 6E). In the colony forming assay, the miR-665 inhibitor significantly repressed the colony forming ability, which was reversed after SRCIN1 silencing in SKOV3 cells (Fig. 6F), suggesting that SRCIN1 is involved in miR-665-mediated cell proliferation of ovarian cancer cells.

Negative correlation between miR-665 expression and SRCIN1 mRNA levels in tumor tissues from patients with ovarian cancer. To study the clinical association between miR-665 and SRCIN1, RT-qPCR was applied for the detection of SRCIN1 mRNA levels in 40 pairs of normal and tumor tissues collected from patients with ovarian cancer. SRCIN1 mRNA levels were significantly decreased in tumor tissues compared with normal tissues (Fig. 7A). Notably, a significant negative correlation was observed between SRCIN1 mRNA levels and miR-665 expression (Fig. 7B).

\section{Discussion}

Due to their involvement in the regulation of sustained cell growth signaling, miRNAs are considered as potential biomarkers and therapeutic targets for cancer (26). By analyzing the comprehensive miRNA profiles of samples from volunteers and patients with ovarian cancer, 10 miRNAs were identified as accurate predictors for the early detection of ovarian cancer (17). Among them, miR-320a was identified as a tumor suppressor of ovarian cancer by targeting twist family bHLH transcription factor 1 and MAPK1 $(27,28)$. In the present study, miR-665 promoted ovarian cancer cell proliferation by targeting SRCIN1, which activated MAPK/ERK signaling.

The role of miR-665 in cell proliferation is cell context dependent. During the development of intervertebral disc degeneration, the miR-665 level gradually increased to repress the expression of growth differentiation factor 5 , thus promoting the cell proliferation of nucleus pulposus cells (29). In osteosarcoma cells, ectopic expression of miR-665 suppressed cell proliferation, migration and invasion by targeting Ras-related protein Rab-23 (30). The expression of miR-665 in tumor tissues and normal tissues from patients with ovarian cancer was analyzed, and was upregulated in tumor tissues compared with the normal tissues, which was also observed in two ovarian cancer cell lines compared with the immortal ovarian cancer cell line. These results of miR-665 are consistent with its role in discriminating patients with non-epithelial ovarian cancer from non-cancer controls (17). Furthermore, inhibition of miR-665 significantly inhibited cell proliferation and colony formation of ovarian cancer cells, which further validated the oncogenic role of miR-665 in ovarian cancer.

MAPK/ERK signaling is a well-studied driver of cancer initiation and development (31). In ovarian cancer, sustained activation of MAPK/ERK signaling is associated with strong cell proliferation, metastasis and stemness ability (32). Dysregulation of positive and negative regulators is responsible for the uncontrolled activation of the MAPK/ERK pathway (33). The present western blotting results showed that the MAPK/ERK pathway was inactivated after miR-665 inhibition, suggesting that miR-665 might promote cell proliferation via activation of MAPK/ERK signaling. 
A
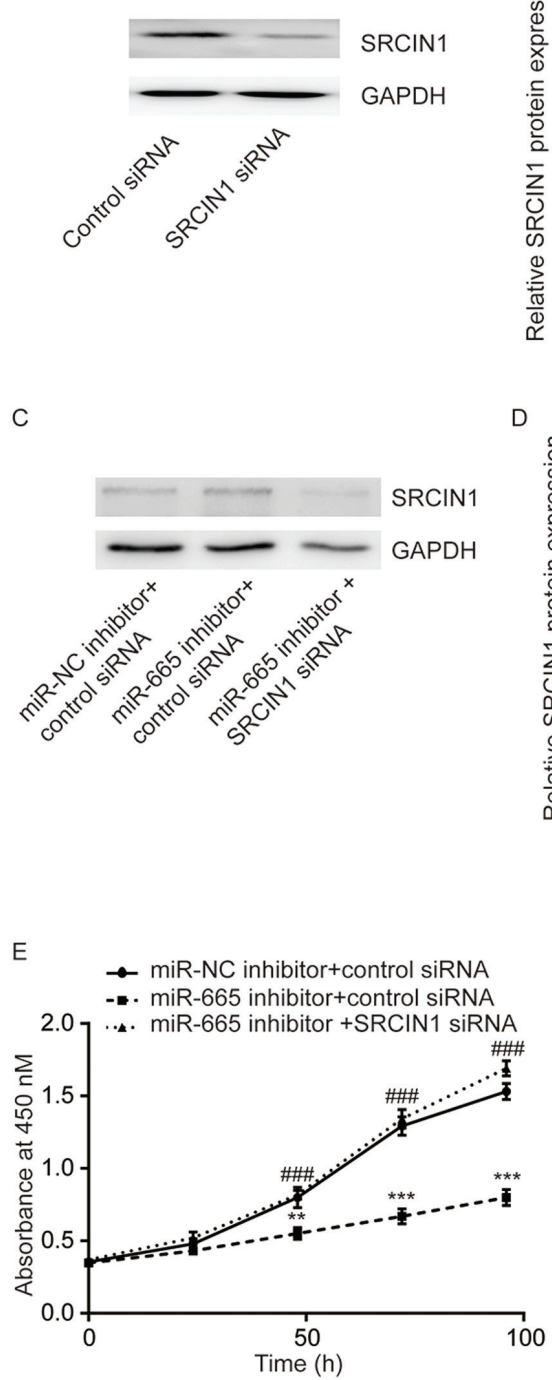
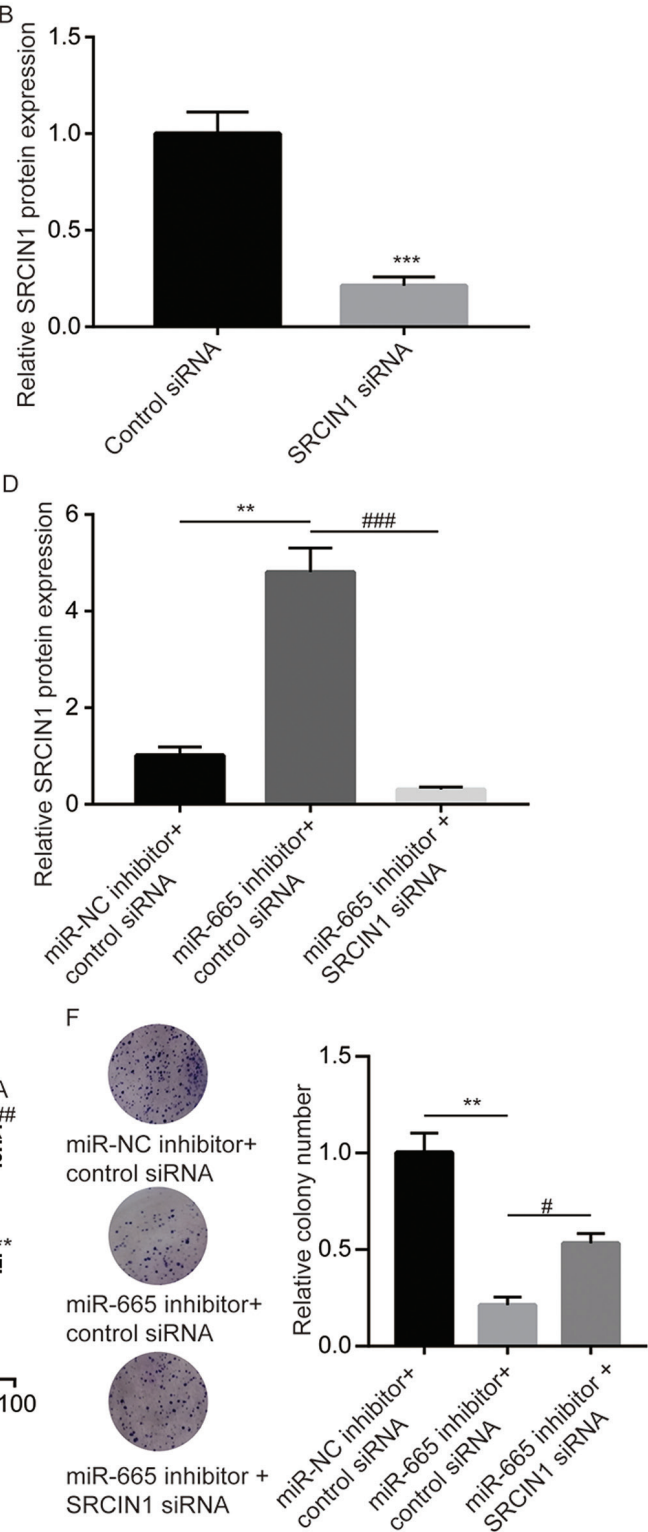

Figure 6. miR-665 regulates cell proliferation mainly through SRCIN1 in ovarian cancer cells. (A) In SKOV3 cells, transfection of SRCIN1 siRNA decreased SRCIN1 protein expression. (B) Quantitative analysis of SRCIN1 expression following transfection with SRCIN1 siRNA. ${ }^{* * *} \mathrm{P}<0.001$ vs. control siRNA. (C) In SKOV3 cells, transfection of miR-665 inhibitor increased the protein level of SRCIN1, which was downregulated after transfection with SRCIN1 siRNA. (D) Quantitative analysis of SRCIN1 expression following multiple transfections. (E) In SKOV3 cells, transfection of miR-665 inhibitor inhibited cell proliferation, which was reversed after transfection SRCIN1 siRNA. (F) In SKOV3 cells, transfection of miR-665 inhibitor inhibited colony formation, which was reversed after transfection with SRCIN1 siRNA. ${ }^{* *} \mathrm{P}<0.01 \mathrm{vs}$. miR-NC inhibitor + control siRNA; ${ }^{* * * *} \mathrm{P}<0.001 \mathrm{vs}$. miR-NC inhibitor + control siRNA, control siRNA; ${ }^{\#} \mathrm{P}<0.05$ vs. miR-665 inhibitor + SRCIN1 siRNA; ${ }^{\# \# "} \mathrm{P}<0.001$ vs. miR-665 inhibitor + SRCIN1 siRNA. miR-665, microRNA-665; miR-NC, microRNA negative control; siRNA, small interfering RNA; SRCIN1, Src kinase signaling inhibitor 1.
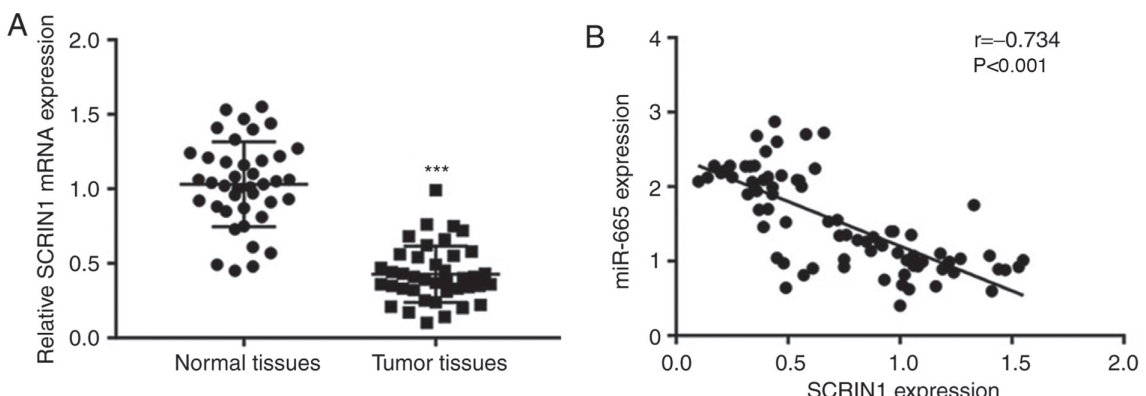

Figure 7. SRCIN1 expression is negatively correlated with miR-665 levels in tumor tissues from patients with ovarian cancer. (A) Reverse transcriptionquantitative PCR showed that the expression level of SCRIN1 was downregulated in tumor tissues compared with normal tissues from 40 patients with ovarian cancer. (B) Pearson's correlation analysis showed that SRCIN1 mRNA levels were negatively correlated with miR-665 levels in tumor tissues from 40 patients with ovarian cancer. ${ }^{* * * *} \mathrm{P}<0.001$ vs. normal tissues. miR-665, microRNA-665; SRCIN1, Src kinase signaling inhibitor 1. 
Among several potential target genes of miR-665 predicted using TargetScan, SRCIN1, a negative regulator of MAPK/ERK signaling, was identified. Previous studies demonstrated that SRCIN1 was involved in the progression of cancer; including gastric cancer and breast cancer; SRCIN1 was targeted and repressed by miR-374a and miR-346, respectively $(34,35)$. The present study demonstrated that miR-665 acted as a new miRNA regulator of SRCIN1 in ovarian cancer, which was verified by the following results: Inhibition of miR-665 increased SRCIN1 at the mRNA and protein levels in ovarian cancer; and miR-665 mimic significantly reduced the luciferase activity of cells transfected with SRCIN1 3'UTR-WT. The present study further demonstrated that silencing of SRCIN1 could reverse miR-665-inhibitor-induced cell growth arrest, suggesting that SRCIN1 may be important for the function of miR-665 in ovarian cancer.

The current study showed overexpression and the role of miR-665 in ovarian cancer in vitro. The molecular mechanism by which miR-665 is aberrantly expressed in ovarian cancer requires further investigation using both in vitro and in vivo models.

In conclusion, the present study showed that miR-665 functioned as an oncogene by targeting SRCIN in ovarian cancer cells, providing rationale for using the miR-665 level as a predictor of ovarian cancer.

\section{Acknowledgements}

Not applicable.

\section{Funding}

No funding was received.

\section{Availability of data and materials}

The datasets used and/or analyzed during the current study are available from the corresponding author on reasonable request.

\section{Authors' contributions}

TX and LY collected the clinical samples. JY designed and supervised the study. PZ, TX, LY and JY acquired and analyzed the data. JY prepared and edited the manuscript. All authors read and approved the final manuscript.

\section{Ethics approval and consent to participate}

All patients provided written informed consent before enrollment in the present study and The Ethic Committee of Xinjiang Medical University approved the present study.

\section{Patient consent for publication}

Not applicable.

\section{Competing interests}

The authors declare that they have no competing interests.

\section{References}

1. Bray F, Ferlay J, Soerjomataram I, Siegel RL, Torre LA and Jemal A: Global cancer statistics 2018: GLOBOCAN estimates of incidence and mortality worldwide for 36 cancers in 185 countries. CA Cancer J Clin 68: 394-424, 2018.

2. Bristow RE: Surgical standards in the management of ovarian cancer. Curr Opin Oncol 12: 474-480, 2000.

3. Harries M and Gore M: Part II: Chemotherapy for epithelial ovarian cancer-treatment of recurrent disease. Lancet Oncol 3: 537-545, 2002.

4. Trimble EL, Wright $\mathrm{J}$ and Christian MC: Treatment of platinum-resistant ovarian cancer. Expert Opin Pharmacother 2: 1299-1306, 2001

5. Chen L, Cheng X, Tu W, Qi Z, Li H, Liu F, Yang Y, Zhang Z and Wang Z: Apatinib inhibits glycolysis by suppressing the VEGFR2/AKT1/SOX5/GLUT4 signaling pathway in ovarian cancer cells. Cell Oncol (Dordr) 42: 679-690, 2019.

6. Bartel DP: MicroRNAs: Genomics, biogenesis, mechanism, and function. Cell 116: 281-297, 2004.

7. Bartel DP: MicroRNAs: Target recognition and regulatory functions. Cell 136: 215-233, 2009.

8. Alvarez-Garcia I and Miska EA: MicroRNA functions in animal development and human disease. Development 132: 4653-4662, 2005.

9. Ma J, Li Y, Yao L and Li X: Analysis of MicroRNA expression profiling involved in MC-LR-induced cytotoxicity by high-throughput sequencing. Toxins (Basel) 9: E23, 2017.

10. Ma J and Li X: High-throughput sequencing provides an insight into the hepatotoxicity mechanism of MC-LR in HepG2 cells. Toxin Reviews 37: 1-10, 2017.

11. Zhang L, Volinia S, Bonome T, Calin GA, Greshock J, Yang N, Liu CG, Giannakakis A, Alexiou P, Hasegawa K, et al: Genomic and epigenetic alterations deregulate microRNA expression in human epithelial ovarian cancer. Proc Natl Acad Sci USA 105: 7004-7009, 2008.

12. Iorio MV, Visone R, Di Leva G, Donati V, Petrocca F, Casalini P, Taccioli C, Volinia S, Liu CG, Alder H, et al: MicroRNA signatures in human ovarian cancer. Cancer Res 67: 8699-8707, 2007.

13. Davidson B, Tropé CG and Reich R: The clinical and diagnostic role of microRNAs in ovarian carcinoma. Gynecol Oncol 133: 640-646, 2014.

14. Xu YZ, Xi QH, Ge WL and Zhang XQ: Identification of serum microRNA-21 as a biomarker for early detection and prognosis in human epithelial ovarian cancer. Asian Pac J Cancer Prev 14: 1057-1060, 2013.

15. Cappellesso R, Tinazzi A, Giurici T, Simonato F, Guzzardo V, Ventura L, Crescenzi M, Chiarelli S and Fassina A: Programmed cell death 4 and microRNA 21 inverse expression is maintained in cells and exosomes from ovarian serous carcinoma effusions. Cancer Cytopathol 122: 685-693, 2014.

16. Xie Z, Cao L and Zhang J: miR-21 modulates paclitaxel sensitivity and hypoxia-inducible factor- $1 \alpha$ expression in human ovarian cancer cells. Oncol Lett 6: 795-800, 2013.

17. Yokoi A, Matsuzaki J, Yamamoto Y, Yoneoka Y, Takahashi K, Shimizu H, Uehara T, Ishikawa M, Ikeda SI, Sonoda T, et al: Integrated extracellular microRNA profiling for ovarian cancer screening. Nat Commun 9: 4319, 2018.

18. Liu W, Yue F, Zheng M, Merlot A, Bae DH, Huang M, Lane D, Jansson P, Lui GY, Richardson V, et al: The proto-oncogene $\mathrm{c}-\mathrm{Src}$ and its downstream signaling pathways are inhibited by the metastasis suppressor, NDRG1. Oncotarget 6: 8851-8874, 2015.

19. Fang D, Chen H, Zhu JY, Wang W, Teng Y, Ding HF, Jing Q, $\mathrm{Su}$ SB and Huang S: Epithelial-mesenchymal transition of ovarian cancer cells is sustained by Racl through simultaneous activation of MEK1/2 and Src signaling pathways. Oncogene 36: 1546-1558, 2017.

20. Le XF and Bast RC Jr: Src family kinases and paclitaxel sensitivity. Cancer Biol Ther 12: 260-269, 2011.

21. Zhang LQ, Lv RW, Qu XD, Chen XJ, Lu HS and Wang Y: Aloesin suppresses cell growth and metastasis in ovarian cancer SKOV3 cells through the inhibition of the MAPK signaling pathway. Anal Cell Pathol (Amst) 2017: 8158254, 2017.

22. Kennedy S, Clynes M, Doolan P, Mehta JP, Rani S, Crown J and O'Driscoll L: SNIP/p140Cap mRNA expression is an unfavourable prognostic factor in breast cancer and is not expressed in normal breast tissue. Br J Cancer 98: 1641-1645, 2008. 
23. Gregoire L, Rabah R, Schmelz EM, Munkarah A, Roberts PC and Lancaster WD: Spontaneous malignant transformation of human ovarian surface epithelial cells in vitro. Clin Cancer Res 7: 4280-4287, 2001.

24. Livak KJ and Schmittgen TD: Analysis of relative gene expression data using real-time quantitative PCR and the 2(-Delta Delta C(T)) method. Methods 25: 402-408, 2001.

25. Fogh J, Wright WC and Loveless JD: Absence of HeLa cell contamination in 169 cell lines derived from human tumors. J Natl Cancer Inst 58: 209-214, 1977.

26. Pal MK, Jaiswar SP, Dwivedi VN, Tripathi AK, Dwivedi A and Sankhwar P: MicroRNA: A new and promising potential biomarker for diagnosis and prognosis of ovarian cancer. Cancer Biol Med 12: 328-341, 2015.

27. Li C, Duan P, Wang J, Lu X and Cheng J: miR-320 inhibited ovarian cancer oncogenicity via targeting TWIST1 expression. Am J Transl Res 9: 3705-3713, 2017.

28. Xu Y, Hu J, Zhang C and Liu Y: MicroRNA320 targets mitogenactivated protein kinase 1 to inhibit cell proliferation and invasion in epithelial ovarian cancer. Mol Med Rep 16: 8530-8536, 2017.

29. Tan H, Zhao L, Song R, Liu Y and Wang L: microRNA-665 promotes the proliferation and matrix degradation of nucleus pulposus through targeting GDF5 in intervertebral disc degeneration. J Cell Biochem 119: 7218-7225, 2018.

30. Dong C, Du Q, Wang Z, Wang Y, Wu S and Wang A: MicroRNA-665 suppressed the invasion and metastasis of osteosarcoma by directly inhibiting RAB23. Am J Transl Res 8 . 4975-4981, 2016.
31. Samatar AA and Poulikakos PI: Targeting RAS-ERK signalling in cancer: Promises and challenges. Nat Rev Drug Discov 13: 928-942, 2014

32. Yu Z, Ye S, Hu G, Lv M, Tu Z, Zhou K and Li Q: The RAF-MEK-ERK pathway: Targeting ERK to overcome obstacles to effective cancer therapy. Future Med Chem 7: 269-289, 2015.

33. Chen R, Liao JY, Huang J, Chen WL, Ma XJ and Luo XD: Downregulation of SRC kinase signaling inhibitor 1 (SRCIN1) expression by MicroRNA-32 promotes proliferation and epithelial-mesenchymal transition in human liver cancer cells. Oncol Res 26: 573-579, 2018

34. Xu X, Wang W, Su N, Zhu X, Yao J, Gao W, Hu Z and Sun Y: miR-374a promotes cell proliferation, migration and invasion by targeting SRCIN1 in gastric cancer. FEBS Lett 589: 407-413, 2015.

35. Yang F, Luo LJ, Zhang L, Wang DD, Yang SJ, Ding L, Li J, Chen D, Ma R, Wu JZ and Tang JH: miR-346 promotes the biological function of breast cancer cells by targeting SRCIN1 and reduces chemosensitivity to docetaxel. Gene 600: 21-28, 2017.

This work is licensed under a Creative Commons Attribution-NonCommercial-NoDerivatives 4.0 International (CC BY-NC-ND 4.0) License. 